\title{
Estimation of Neural Noise Spectrum in a Postural Control Model
}

\author{
A.F. Kohn \\ Biomedical Engineering Laboratory, Universidade de São Paulo, EPUSP, Brazil
}

\begin{abstract}
A simple linear feedback control model representing a standing human is driven by neural and torque noise sources. A mathematical expression was derived for the neural noise spectrum as a function of spectra computed from two signals: the electromyogram (EMG) and the angle of the subject with respect to the vertical direction. Simulations of a stochastic postural control system were used to generate "EMG" and "angle" signals that were used in the theoretically derived neural noise spectrum. The comparison with the directly estimated neural noise spectrum showed that the mathematical expression yields estimates that have useful information about the spectral shape of the neural noise. In addition, the method also yields useful estimates of the neural noise spectral bandwidth.
\end{abstract}

Keywords- neural noise, postural oscillations, spectral estimation, postural control model.

\section{INTRODUCTION}

During quiet standing, a human being presents random oscillations, which can be quantified by the variations of the angle of the body with respect to the direction of gravity. There are at least two sources for these random oscillations: one due the neuromuscular system ("neural noise") and the other due to endogenous mechanical phenomena ("torque noise"). An important source of neural noise is the synaptic bombardment received by a motoneuron [1], which may cause variations in the times of discharge of the motoneurons and in the recruitment of the motor units from the motoneuron pool. These sources of variability of neural origin cause random variations in the maintained force by a given muscle, which will cause torque variability around a joint.

The sources of direct mechanical variability would be due to movement of internal organs like the heart and the lung, besides eventual involuntary movement of other parts of the body, such as slight arm movements. The latter should be well controlled during an experiment.

Theoretical studies of postural control have included both the torque and neural noises [2-4], even though the features of both are practically unknown in humans, due to the inherent difficulties of noninvasive experiments. An overall objective of this work is to develop a methodology to obtain estimates of the power spectral density of the neural noise when the electromyogram and the angle of the subject with respect to the vertical are measured along time. As a first step towards this goal a simple model of the postural control system is proposed here, and an expression of the neural noise power spectrum is derived in terms of the assumed known elements. The model deals with oscillations in the anteroposterior direction, which are the most relevant when the subject is standing with the feet apart in a relaxed standing position. The model is linear and time-invariant, which is, in a first approximation, compatible with physiology. [2,3]

\section{Model}

The model adopted for the postural control system is shown in Fig. 1. The human being is represented as an inverted pendulum, with rotations occurring around the ankle. The reference angle will be taken as $0^{\circ}$, but any other value could have been adopted, respecting the physiology, without any loss of generality. The central nervous system (CNS) receives spike trains from the sensory receptors that signal the angle of the ankle joint. The variability of this angle is the same as that between the vertical and the axis of the inverted pendulum, which in Fig. 1 is indicated as $\boldsymbol{\theta}$.

The CNS generates commands to the muscle (soleus) that are uncorrelated with the additive noise of neural origin. The neural command is assumed to be approximately equal to the electromyogram (EMG) envelope. The force generated by the muscle results in a torque applied on the inverted pendulum, which is described by a second order unstable system $G_{L}$. Two sources of noise are included: noise generated by the nervous system $\mathbf{v}$, and torque noise $\mathbf{q}$ A basic hypothesis is that both noises are independent, which is physiologically plausible.

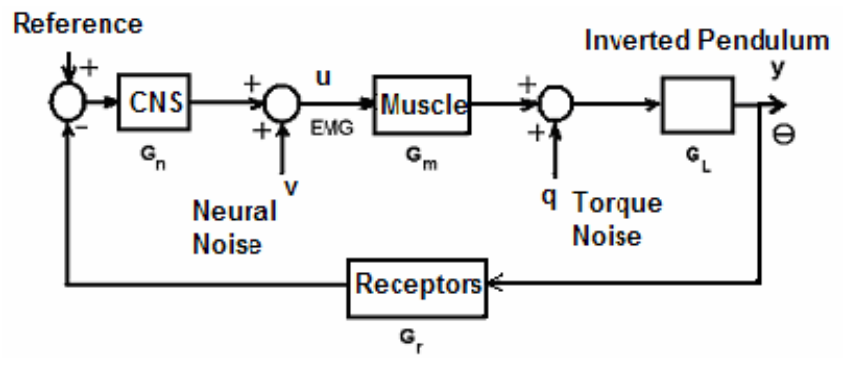

Fig. 1. Model adopted for the postural control system. 
The proposition with respect to Fig. 1 is to estimate $S_{\mathrm{vv}}(j \omega)$, the power spectral density of the neural noise $\mathbf{v}$. It is assumed that the following signals and systems are known: signals $\mathbf{u}$ and $\mathbf{y}$ and the transfer functions of each block $\left(\mathrm{G}_{\mathrm{n}}\right.$, $\mathrm{G}_{\mathrm{m}}, \mathrm{G}_{\mathrm{L}}$ and $\mathrm{G}_{\mathrm{r}}$ ). The expected values of all random signals in the control loop are assumed equal to zero, without loss of generality.

The cross-spectrum $S_{x y}(j \omega)$ of the two random signals $\mathrm{x}(\mathrm{t})$ and $\mathrm{y}(\mathrm{t})$ will be taken as the Fourier transform of $\mathrm{R}_{\mathrm{xy}}(\tau)=\mathrm{E}[\mathrm{X}(\mathrm{t}+\tau) \mathrm{Y}(\mathrm{t})]$. Keeping the consistency with this notation and to make the math easier to develop, $S_{x y}(j \omega)$ will be taken as the limit as $T \rightarrow \infty$ of

$$
\frac{1}{T} \overline{X(j \omega) Y^{*}(j \omega)}
$$

where $X(j \omega)$ is the Fourier transform of a segment of $x(t)$ of finite duration $\mathrm{T}$ and the bar over the spectral product indicates the expected value. The derivations will use $\mathrm{X}$ instead $X(j \omega)$, for example, to ease the notation. The finite Fourier transforms will be indicated by the corresponding capital letter to that of the random signal. The average and limit operations will be taken only at the end.

\section{THE NEURAL NOISE POWER SPECTRUM}

From Fig. 1:

$$
U=V-G_{n} G_{r} Y
$$

and

$$
Y=G_{L} Q+G_{L} G_{M} U
$$

Substituting (2) in (1):

$$
U=V-G_{n} G_{r} G_{L} Q-G_{n} G_{r} G_{L} G_{m} U
$$

and hence:

$$
U=\frac{V-G_{n} G_{r} G_{L} Q}{1+G_{n} G_{r} G_{L} G_{m}}
$$

Multiplying equation (1) by $\mathrm{Y}^{*}$ :

$$
U Y^{*}=V Y^{*}-G_{n} G_{r} Y Y^{*}
$$

Substituting (2) in (4), computing the averages and then the limit for $T \rightarrow \infty$, one has

$$
S_{u y}=G_{L}^{*} S_{v q}+G_{L}^{*} G_{m}^{*} S_{v u}-G_{n} G_{r} S_{y y}
$$

In equation (5) one should notice that $S_{v q}$ is equal to zero, by hypothesis. For more compactness the following definition will be used:

$$
G_{M A}=G_{n} G_{r} G_{L} G_{m}
$$

The product of $\mathrm{V}$ with the complex-conjugate of equation (3) gives:

$$
V U^{*}=\frac{V V^{*}-G_{n}^{*} G_{r}^{*} G_{L}^{*} V Q^{*}}{1+G_{M A}^{*}}
$$

Computing the averages and then the limit for $T \rightarrow \infty$ in (7), and remembering that the neural noise is assumed independent from the torque noise, we have

$$
S_{v u}=\frac{S_{v v}}{1+G_{M A}^{*}}
$$

The expression for $S_{v u}$ in (8) will be substituted into equation (5), resulting in:

$$
S_{u y}=\frac{G_{L}^{*} G_{m}^{*} S_{v v}}{1+G_{M A}^{*}}-G_{n} G_{r} S_{y y}
$$

From (9), $S_{v v}$ may be isolated as

$$
S_{v v}=\left[S_{u y}+G_{n} G_{r} S_{y y}\right] / G
$$

where

$$
G=\frac{G_{L}^{*} G_{m}^{*}}{1+G_{M A}^{*}}
$$

Another expression, given below is equivalent to (10) but shows every frequency response function shown in Fig. 1:

$$
S_{v v}=\left[S_{y u}+G_{n}^{*} G_{r}^{*} S_{y y}\right] \cdot\left[\frac{1+G_{n} G_{r} G_{L} G_{m}}{G_{L} G_{m}}\right]
$$

From (12) it may be seen that an expression for the neural noise power spectrum was obtained as a function of $\mathbf{i}$ the cross-spectrum of the angle $\theta$ and the EMG envelope and ii the auto-spectrum of the angle $\theta$. These two spectra may be estimated without difficulties from the angle and EMG measured during a typical experiment of postural control. In an experimental situation with human subjects, the transfer functions of some of the blocks would have to be identified, which is out of the scope of this paper. 


\section{Simulations}

Expression (12) shall be used to estimate the neural noise power spectrum from simulations of the system of Fig. 1. The simulations were implemented in Simulink (Mathworks, USA) based on the diagram shown in Fig. 2.

Block $\mathrm{G}_{\mathrm{n}}$ Is a PD (proportional/derivative) controller, as usually assumed for postural control systems [4]. The neural noise $\mathbf{v}$ is obtained applying white noise to the filter given by $\mathrm{H}_{1}$. The torque generation by the soleus muscle was modelled from the experimental curves obtained by Bawa e Stein [5] and is represented by the second order system $\mathrm{G}_{\mathrm{m}}$.

The human subject in the standing position is modelled by an inverted pendulum $\left(\mathrm{G}_{\mathrm{L}}\right)$, with parameters usually employed in the literature $[2,3]$.

The feedback $\left(\mathrm{G}_{\mathrm{r}}\right)$ was adopted as a constant gain, in part with the assumption that the sensory receptors respond to a wide frequency range. This is also a simplification usually found in the literature [2]. Finally, the torque noise is also modelled as a white noise filtered by a system $\left(\mathrm{H}_{2}\right)$. The numerical integration was the Dormand-Prince of fifth order, with a fixed step equal to $0.01 \mathrm{~s}$. Each simulation generated $800 \mathrm{~s}$ of random signals from which the spectra needed in expression (12) were computed. As the neural noise signal was available directly from the simulation, it was used to obtain a direct spectral estimation so it is possible to evaluate the quality of the spectrum estimated by (12).

In a first phase, we used only the neural noise, i.e., the torque noise was made equal to zero. Fig. 3a shows the estimated spectrum from $800 \mathrm{~s}$ signals $\mathrm{u}$ and $\mathrm{y}$ based on expression (12). Fig. $3 \mathrm{~b}$ shows the neural noise spectrum obtained directly from the noise signal $\mathbf{v}$. This noise was generated by filtering white noise by a second order filter with a resonance peak $\left(\mathrm{H}_{1}(\mathrm{~s})=1100 /\left(\mathrm{s}^{2}+0,4 \mathrm{~s}+100\right)\right.$.

The spectra in Fig. 3 were normalized so that the regions around $5 \mathrm{~Hz}$ would have a similar amplitude. There is a good reproducibility of the spectral shape using (12), but there is an error around $3 \mathrm{~dB}$ at frequencies below $1 \mathrm{~Hz}$.

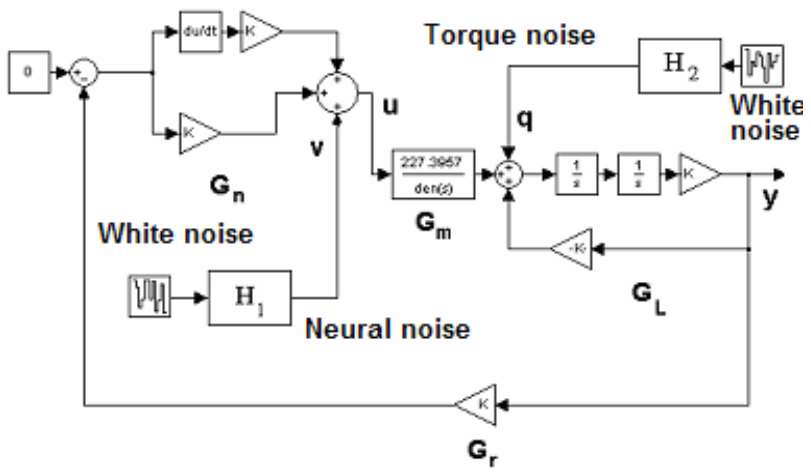

Fig. 2. Simulation diagram of the postural control system.

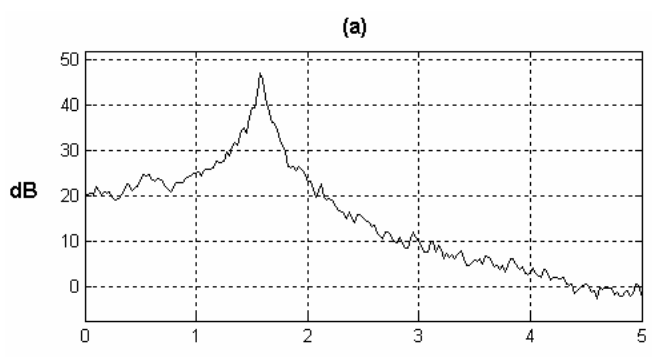

(b)

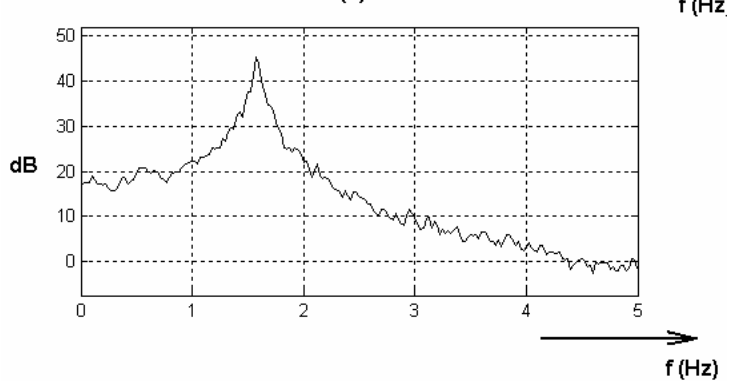

Fig. 3. Neural noise spectra: (a) estimated from (12), (b) estimated directly from $\mathbf{v}$. Torque noise was zero.

Modifying the neural noise spectrum by changing the filter to a first order lowpass system $\left(\mathrm{H}_{1}(\mathrm{~s})=252 /(100 \mathrm{~s}+1)\right)$, the spectra of Fig. 4 were obtained. Again, there is a good reproducibility of the spectral shape, but there is an error of 3 $\mathrm{dB}$ at $1 \mathrm{~Hz}$ and $3,75 \mathrm{~dB}$ at $0,2 \mathrm{~Hz}$. In this simulation torque noise was made equal to zero.

(a)

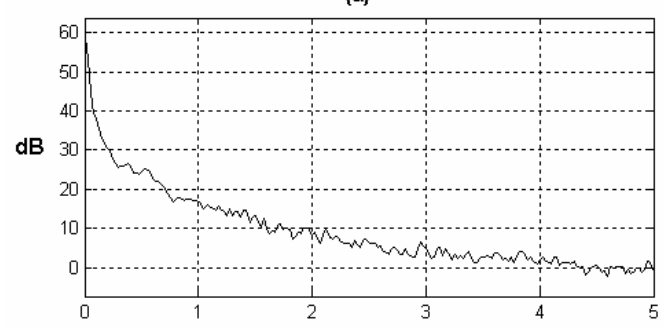

(b) $\mathrm{f}(\mathrm{Hz})$

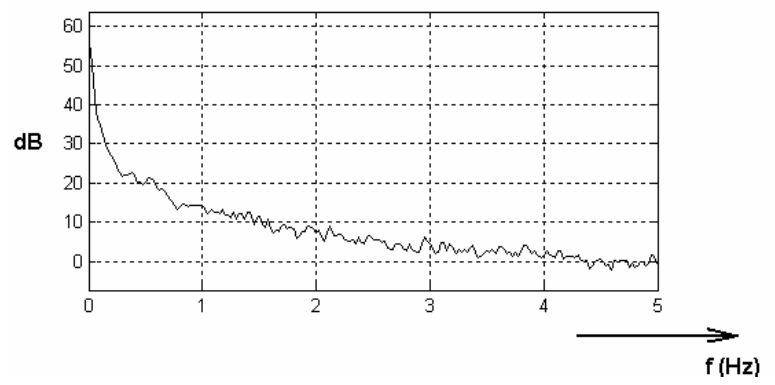

Fig. 4. Neural noise spectra, (a) estimated by (12); (b) directly from $\mathbf{v}$. Torque noise was zero. 
(a)

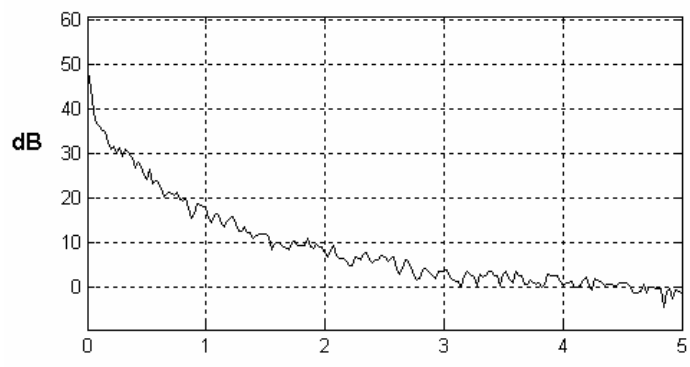

(b)

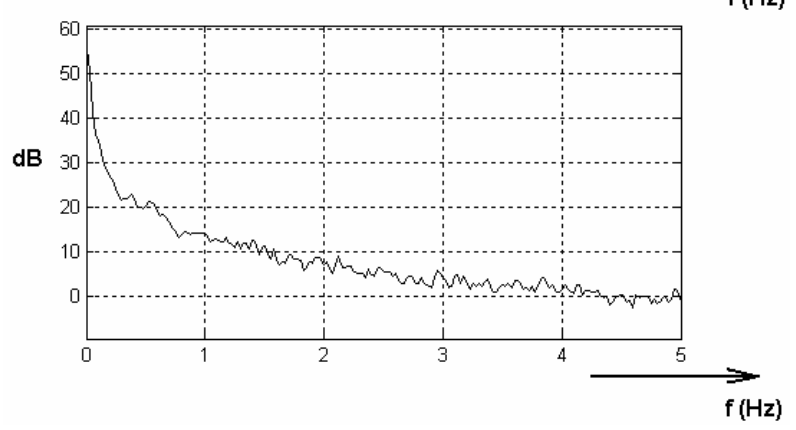

Fig. 5. Neural noise spectra, (a) estimated by (12);

(b) directly from $\mathbf{v}$. Torque noise $\neq 0$.

Next, both noise sources were included in the simulation. The neural noise had the same spectrum as in Fig. $4 \mathrm{~b}$ and the torque noise came from filtering white noise by $\mathrm{H}_{2}(\mathrm{~s})=10 /(\mathrm{s}+1)$. The standard deviation of the output $\mathrm{y}$ was 7.1467e-5 (3.6391e-5) when only the neural (torque) noise were applied. The spectrum estimated from (12) is seen in Fig. 5a and the direct spectral estimation is seen in Fig. 5b. The shape is well reproduced but the low frequency errors are larger than the cases without simultaneous torque noise. For example, the error at $1 \mathrm{~Hz}$ was $4 \mathrm{~dB}$ and at $0,2 \mathrm{~Hz}$ it was $4,12 \mathrm{~dB}$.

The filters $\mathrm{H}_{1}(\mathrm{~s})$ and $\mathrm{H}_{2}(\mathrm{~s})$ in these simulations, when they were lowpass, were chosen similarly to those found in the literature $[2,4]$. The choice of a passband filter was only to check for the sensitivity of (12) to the neural noise spectral shape.

\section{Discussion And Conclusion}

The neural noise acting on the postural control system has been postulated in theoretical work [2,3] but no approach to its estimation was found in the literature. Its direct experimental measurement is partially feasible in cats $[1,6]$ but clearly impossible to obtain in humans. Therefore, indi- rect methods have to used, based on the measurement of externally available signals, such as the EMG and the sway angle. In spite of the simplicity of the chosen postural control model and of the assumption of known transfer functions of each subsystem, the approach has its validity as a first step in the goal of estimating the neural noise from real experiments in humans. The simulation results have suggested that, under the adopted hypotheses, it is possible to obtain spectral estimates of the neural noise which are potentially useful for the study of postural control. The estimation errors seem to be acceptably small for the intended applications. Questions such as (i) what is the shape of the neural noise power spectrum, and (ii) what is the frequency range where there is more power in the neural noise, seem feasible to be answered by the presented approach.

The application to real problems will require additional effort, from the signal processing, modeling and system identification points of view. The concepts and initial approach of the present work should serve as a starting point for the much more challenging task ahead.

\section{AcKNOWLEDGMENT}

Project was funded by Fapesp, CNPq and Capes.

\section{REFERENCES}

1. Calvin WH, Stevens CF (1968) Synaptic noise and other sources of randomness in motoneuron interspike intervals. J Neurophysiol 31:574-587

2. Maurer C, Peterka RJ (2005) A new interpretation of spontaneous sway measures based on a simple model of human postural control. J Neurophysiol 93:189-200

3. Peterka RJ (2000) Postural control model interpretation of stabilogram diffusion analysis. Biol Cybern 82:335-343

4. Masani K et al (2003) Importance of body sway velocity information in controlling ankle extensor activities during quiet stance. J Neurophysiol 90:3774-3782

5. Bawa P, Stein RB (1976) Frequency responses of human soleus muscle. J Neurophysiol 39:788-793

6. Manjarrez E, Hernandez-Paxtian ZJ, Kohn AF (2005) A spinal source for the synchronous fluctuations of bilateral monosynaptic reflexes in cats. J Neurophysiol 94: 3199-3210

$\begin{array}{ll}\text { Author: } & \text { André Fabio Kohn } \\ \text { Institute: } & \text { Universidade de Sao Paulo, LEB, EPUSP } \\ \text { Street: } & \text { Cx.P. 61548 CEP 05424-970 } \\ \text { City: } & \text { São Paulo } \\ \text { Country: } & \text { Brazil } \\ \text { Email: } & \text { andfkohn@leb.usp.br }\end{array}$

